\title{
Novas tecnologias aplicadas à pesquisa em História da Educação
}

New technologies applied to research in History of Education

Nuevas tecnologías aplicadas a la investigación en Historia de la Educación

\author{
Fabio Gomes Rocha \\ Universidade Tiradentes (Brasil) \\ http://lattes.cnpq.br/3161449561865597 \\ http://orcid.org/0000-0002-0512-5406 \\ gomesrocha@gmail.com
}

\author{
Ester Fraga Vilas-Bôas Carvalho do Nascimento \\ Universidade Tiradentes (Brasil) \\ http://lattes.cnpq.br/5387293048319734 \\ https://orcid.org/0000-0002-4050-767X \\ ester_fraga@unit.br
}

\section{Resumo}

O emprego de novos recursos para suporte à pesquisa histórica é uma imposição da atualidade aos investigadores do campo da História da Educação, não devendo se limitar à aplicação técnica, mas se estender ao exame de processos tecnológicos que venham a potencializar a exploração de fontes. Nesse contexto, o presente trabalho, teve como objetivo caracterizar o estado do conhecimento acerca das novas tecnologias empregadas nas pesquisas de História da Educação, a partir das publicações disponíveis no Portal de periódicos e no BDTD CAPES. Buscou-se verificar quais são as tecnologias empregadas e o contexto de sua aplicação. Os resultados indicaram que a digitalização tem sido o recurso mais utilizado nos trabalhos dos pesquisadores desse campo. Constatou-se, ainda, que a disponibilização dos documentos coletados ocorre, com maior frequência, por meio de CD/DVD. Com menor incidência, os autores investiram na elaboração de banco de dados e repositórios para a distribuição de conteúdos.

Palavras-chave: Digitalização. História da Educação. Novas tecnologias. Repositório digital. 


\begin{abstract}
The use of new resources to support historical research is an imposition of the present time for researchers in the field of History of Education, not limited to technical application, but extends to the examination of technological processes that will enhance the exploration of sources. In this context, the purpose of this exploratory and descriptive study was to characterize the state of knowledge about the new technologies used in research on the History of Education, based on the publications available in the Journal Portal and the BDTD CAPES. It was sought to verify which technologies are used and the context of their application. The results indicated that the digitization has been the most used resource in the work of researchers in this field. It was also verified that the availability of the collected documents occurs, more frequently, through CD/DVD. With less incidence, the authors invested in the elaboration of database and repositories for the distribution of contents.
\end{abstract}

Keywords: Scanning. History of Education. New technologies. Digital Repository.

\title{
Resumen
}

El empleo de nuevos recursos para apoyar la investigación histórica es una imposición de la actualidad a los investigadores del campo de la Historia de la Educación, no debiendo limitarse a la aplicación técnica, sino extenderse al examen de procesos tecnológicos que puedan potenciar la explotación de fuentes. En este contexto, el presente trabajo, de carácter exploratorio y descriptivo, tuvo como objetivo caracterizar el estado del conocimiento acerca de las nuevas tecnologías empleadas en las investigaciones de Historia de la Educación, a partir de las publicaciones disponibles en el Portal de periódicos y en el BDTD CAPES. Se buscó verificar cuáles son las tecnologías empleadas y el contexto de su aplicación. Los resultados indicaron que la digitalización ha sido el recurso más utilizado en los trabajos de los investigadores de ese campo. Se constató, además, que la puesta a disposición de los documentos recogidos ocurre, con mayor frecuencia, por medio de CD/DVD. Con menor incidencia, los autores invirtieron en la elaboración de bases de datos y repositorios para la distribución de contenidos.

Palavras-clave: Digitalización. Historia de la Educación. Nuevas tecnologías. Repositorio digital. 


\section{Introdução}

A produção intelectual brasileira, segundo Amorim (2000), é prejudicada pela desorganização dos órgãos de documentos. Além disso, bases dispersas inviabilizam ou, no mínimo, dificultam a descoberta de informações relevantes ao trabalho do pesquisador. Assim, o emprego de novas tecnologias para o trato documental pode subsidiar pesquisas históricas no tocante à conservação dos dados, consulta integrada e exploração de novos conhecimentos em base de dados (AMORIM, 2000).

O emprego de novos recursos para suporte à pesquisa histórica, segundo Gondra (2000), é uma imposição da atualidade aos investigadores do campo da História da Educação, não devendo se limitar à aplicação técnica, mas se estender ao exame de processos tecnológicos que venham a potencializar a exploração de fontes. Disso, depreende-se a necessidade de uma relação com outros campos disciplinares, focalizando eventuais contribuições para o acesso, análise e disponibilização de materiais ou acervos documentais. Neste sentido, Bonato (2011) afirma que o campo da História da Educação amplia-se nas suas possibilidades de pesquisa por meio das novas tecnologias, visto que elas podem fornecer novos suportes a registro, armazenamento e recuperação de informações. Observa-se, assim, o necessário diálogo do campo com a Arquivologia e a Tecnologia da Informação e Comunicação.

Nesse contexto, o presente trabalho, de cunho exploratório e descritivo, tem como objetivo caracterizar o estado do conhecimento acerca das novas tecnologias empregadas nas pesquisas de História e de História da Educação, a partir das publicações disponíveis no Portal de periódicos e no Banco de Teses da Coordenação de Aperfeiçoamento de Pessoal de Ensino Superior (CAPES). Para o norteamento da busca nessas fontes foram definidas as seguintes questões de pesquisa: a) quais são as tecnologias empregadas?; b) em qual contexto a tecnologia foi empregada? As respostas a essas indagações poderão auxiliar pesquisadores na adequada seleção de tecnologias a serem empregadas em suas investigações.

O trabalho está organizado, a partir dessa seção introdutória, da seguinte forma: na seção 2 é apresentada fundamentação teórica, na seção 3 é exposta a metodologia adotada, na seção 4 são apresentados e discutidos os resultados e, por fim, na seção 5 apresentam-se as conclusões sobre o estudo.

\section{Fundamentação teórica}

A História da Educação como disciplina surgiu no final do século XIX, mas não como uma área da História, mas, sim, da própria área da Educação (GALVÃO, LOPES, 2011). No Brasil, a História da Educação foi introduzida no currículo da escola normal a partir de 1928 e a sua evolução culminou na criação do Grupo de Trabalho de História da Educação da Associação Nacional de Pós-Graduação e Pesquisa em Educação, em 1984 (ANPED, 2018). Essa ação proporcionou uma ampliação no campo de pesquisa, gerando mudanças significativas como as melhorias nos contornos conceituais e metodológicos, bem como a ampliação das possibilidades sobre os objetos e as fontes. Entre as tendências teóricometodológicas adotadas destaca-se a História Cultural que, segundo Chartier (1990) aponta para a necessidade de estudo sobre a materialidade de objetos culturais, considerando-se a forma de produção, circulação, consumo e apropriações.

Sob esse olhar, as fontes, matéria-prima do historiador, surgem como meio enriquecedor para o conhecimento do contexto que a envolve. Em sua busca de elementos que façam emergir evidências sobre o objeto investigado, o pesquisador deve, então, considerar o maior número de fontes possíveis, questionando-as e relacionando-as de forma a obter a proximidade mais possível do cenário investigado, diante das comprovações encontradas. Como menciona Tuchman (1991, p.11): "Não podemos nunca ter certeza de ter recapturado o passado tal como 
realmente foi. Mas o mínimo que podemos fazer é ficar dentro das provas".

Cabe ressaltar que, no âmbito das fontes documentais, Le Goff (1996) alerta que o documento não é um retrato da realidade, mas um resultado de escolhas conscientes ou inconscientes, tanto na sua etapa de elaboração como na de preservação. Toma-se conhecimento do passado com base nos documentos preservados, os quais foram escritos por pessoas que ali aplicaram os seus pontos de vista. Em seu conceito de documentomonumento, Le Goff (1996) menciona:

O documento não é inócuo. É, antes de mais nada, o resultado de uma montagem, consciente ou inconsciente, das sociedades que o produziram, mas também das épocas sucessivas durantes as quais continuou a viver, talvez esquecido, durante as quais continuou a ser manipulado, ainda que pelo silêncio. O documento é uma coisa que fica, que dura, e o testemunho, o ensinamento (para evocar a etimologia) que ele traz devem ser em primeiro lugar analisados, desmitificando-lhe o seu significado aparente. O documento é monumento. Resulta do esforço das sociedades históricas para impor ao futuro - voluntária ou involuntariamente - determinada imagem de si próprias. No limite, não existe um documento verdade. Todo documento é mentira. Cabe ao historiador não fazer o papel de ingênuo. Os medievalistas, que tanto trabalharam para construir uma crítica sempre útil, decerto - do falso, devem superar essa problemática, porque qualquer documento é, ao mesmo tempo, verdadeiro - incluindo talvez sobretudo os falsos - e falso, porque um monumento é em primeiro lugar uma roupagem, uma aparência enganadora, uma montagem. É preciso começar por desmontar, demolir esta montagem, desestruturar esta construção e analisar as condições de produção dos documentosmonumentos. (LE GOFF, 1996. p.538).

A busca pelas "condições de produção", indicada por Le Goff (1996), impele o historiador a considerações de fontes adicionais que elucidem uma situação inicialmente dada como fato documental. O exame apurado sobre essas fontes diversas impõe o uso de recursos que possibilitem a identificação do maior número de evidências que se relacionem ao cenário e objeto investigado. Para isso, é necessário que o pesquisador adote tecnologias que permitam armazenar, catalogar e consultar fontes de investigação.

Tais tecnologias tiveram a sua utilização multiplicada pelos historiadores partir da década de 1980 (LOMBARDI, 2000). Alguns dos fatores que contribuíram para isso foi o barateamento dos equipamentos, e a ampliação do uso dos microcomputadores, surgidos na década de 1970, nos Estados Unidos, e no Brasil, em 1974, com o computador "Cobra". Esse equipamento foi uma evolução do projeto "Pato Feio", de 1972, o qual envolveu uma equipe com membros da Universidade de São Paulo (USP), Pontifícia Universidade Católica do Rio de Janeiro (PUCRJ) e Serviço Federal de Processamento de Dados (SERPRO). Essa equipe criou o primeiro computador comercial, totalmente projetado e construído no Brasil (WAZLAWICK, 2016), tornando-se popular, principalmente, entre empresas públicas e Universidades. A partir da década de 1990, o computador passou a ser utilizado também pelas empresas privadas, consolidando as práticas informatizadas nos negócios. $\mathrm{O}$ uso de computadores e o alto volume de dados gerados promoveram o surgimento da Internet, em 1992. Inicialmente com utilização não comercial, restrita ao âmbito acadêmico e militar, a Internet evoluiu a partir da criação dos navegadores Mosaic, em 1993, e Netscape, em 1994 (WAZLAWICK, 2016). Com esse novo recurso, a Internet passou a ser utilizada por usuários não técnicos e pesquisadores de diversos campos, entre eles os da História da Educação. 
A relevância das novas tecnologias para a História da Educação já foi alvo de diversas discussões, destacando-se o debate ocorrido em agosto de 2000, promovido pelo Grupo de Trabalho História da Educação da ANPEd, durante o seminário "O impacto das novas tecnologias na pesquisa e na formação do pesquisador em História da Educação", que resultou na publicação da obra "Arquivos, fontes e novas tecnologias: questões para a história da educação" (FARIA FILHO, 2000). Dessa forma, autores do campo, como Gondra (2000) e Fonseca (2000) e Lombardi (2000) passaram a indicar que essa é uma imposição da atualidade para os pesquisadores da História da Educação, os quais devem se apropriar e se beneficiar dos recursos proporcionados pelas novas tecnologias.

\section{Metodologia}

A metodologia adotada considerou as características e procedimentos da pesquisa aplicada, que em sua finalidade visa à obtenção de conhecimentos direcionados a uma situação específica (BOAVENTURA, 2004; KOTHARI, 2004; GIL, 2010; PERDIGÃO; HERLINGER; WHITE,2012). Em seus objetivos, a investigação classifica-se como exploratória e descritiva, permitindo o levantamento de informações sobre o objeto - as novas tecnologias aplicadas à História da Educação, e a identificação de suas características e condições nas produções pesquisadas (SEVERINO,2007; GIL, 2010; HABIB, PATHIK, MARYAM, 2014). Como estratégia de pesquisa foram realizadas buscas no Portal de periódicos e no Banco de Teses da CAPES, identificando-se trabalhos publicados que empregassem novas tecnologias no contexto da História ou História da Educação, e verificando-se o objetivo do uso desses recursos nas respectivas pesquisas. $\mathrm{O}$ marco final estabelecido para as publicações foi o mês de maio de 2018. Os termos empregados para as buscas foram: tecnologia digital, novas tecnologias, história e história da educação. Essas palavras foram organizadas da seguinte forma: (("tecnologia digital" OR "novas tecnologias") AND ("história da educação" OR "história")). Após a busca, foi realizada a leitura do título e resumo para uma seleção inicial. Considerou-se nessa ação o alinhamento dos trabalhos ao objetivo da presente investigação.

A partir de uma seleção inicial de trabalhos com base nos títulos e resumos, procedeuse a leitura para a averiguação e validação diante dos interesses desta pesquisa. A seguir, foram extraídos os dados relativos ao tipo de tecnologia e o contexto de sua aplicação. Após a organização dos dados obtidos, analisou-se os resultados, os quais foram discutidos sob abordagem qualitativa, viabilizando a compreensão das informações que emergiram no âmbito específico das novas tecnologias aplicadas (BOAVENTURA, 2004; BLANCHE, DURRHEIM, PAINTER, 2006; SEVERINO, 2007).

\section{Resultados e discussão}

A partir dos critérios adotados na busca dos trabalhos, obteve-se como resultado a identificação de dezoito produções que apresentavam, no título ou no resumo, a aplicação de novas tecnologias em pesquisa no campo de História ou História da Educação. Diante das questões que nortearam esta investigação constatou-se que a tecnologia mais empregada pelos pesquisadores é a digitalização, encontrada em quinze trabalhos. O foco dos autores é o uso da digitalização para a preservação documental.

Considerando que um trabalho pode abordar a aplicação de mais de uma tecnologia, identificou-se dez produções em que seus autores implementaram bases de dados. Ressalta-se que essa é uma ação relevante para a consolidação de repositórios de armazenamento e catalogação de informações, facilitando o desenvolvimento de novas investigações ou ampliando as já existentes. Para o armazenamento, preservação e distribuição de conteúdos identificaram-se nove trabalhos que utilizaram as mídias CD ou DVD. Com menor número de 
trabalhos, o site, como tecnologia que também permite a distribuição de conteúdos, foi abordado em sete produções. A seguir, constatou-se o uso de processamento de linguagem natural (PLN) em um trabalho e, por fim, apenas uma produção com proposta desenvolvida para um museu virtual. A seguir, apresenta-se a representação desses dados em relação ao total de trabalhos investigados.

Gráfico 1 - Tecnologias utilizadas em artigos de História ou História da Educação.

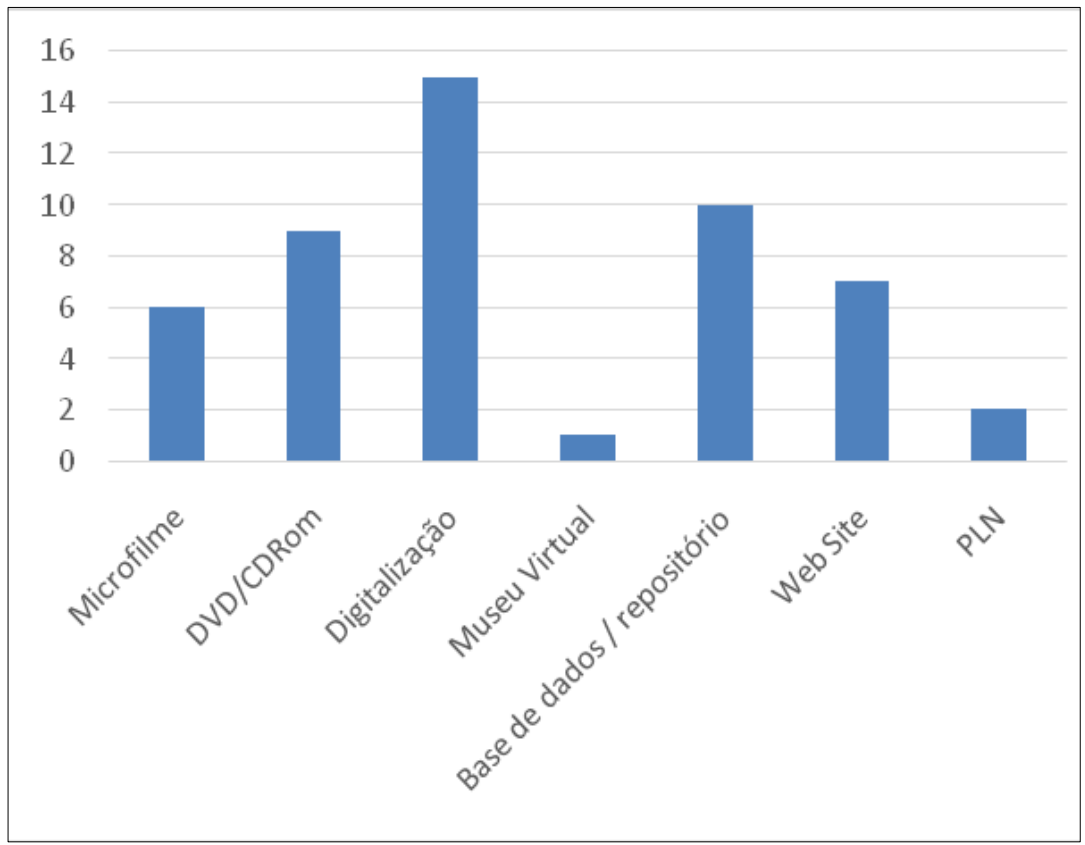

Fonte: Os autores, dados da pesquisa (2018).

Em relação ao contexto em que a tecnologia esteve empregada nas produções, identificou-se que há maior tendência de uso para a preservação documental e redução de espaço físico para o armazenamento. No trabalho de Vieira (2011), que trata sobre Arquivo Público do Estado do Paraná, são apresentados os avanços que microfilmagem e a digitalização de documentos públicos podem trazer aos pesquisadores da História da Educação. Já no trabalho de Bonato (2004) têm-se a abordagem sobre a efetiva contribuição das novas tecnologias para a ampliação e diversificação de fontes nas pesquisas de História da Educação. No entanto, essa autora expõe a necessidade de atenção sobre o acompanhamento dos meios utilizados para o armazenamento. Segundo Bonato (2004), é importante que seja realizada a transferência da informação para novos suportes, sob o risco de, em um futuro próximo, não ser possível obter o acesso a ela devido a um formato tecnológico obsoleto.

Em se tratando de preservação, o trabalho de Pena e Silva (2008) apresenta a digitalização documental como forma de proteger documentos históricos. Para uma gestão eficaz dessa documentação, as autoras propõem uma metodologia para implementação de um sistema de gestão eletrônica de documentos (GED), o que promove a democratização no acesso das informações on-line. $\mathrm{O}$ foco sobre digitalização documental também foi identificado na produção de Lopes et al. (2016). Esses autores descrevem o seu trabalho na criação de um DVD com fontes para a História da Educação de Ouro Preto do Oeste, Estado de Rondônia, contendo vídeos de sessenta e quatro entrevistas, além de outras fontes documentais e iconográficas. $\mathrm{O}$ DVD visou permitir uma preservação da História da Educação, muitas vezes desconhecida, sobre aquela cidade. Ainda voltado para a preservação histórica, o trabalho de Cabral (2002) apresenta o processo de digitalização como forma de conservar e preservar materiais informativos em bibliotecas e arquivos, salientando a importância do planejamento para a 
adoção das novas tecnologias e apresentando como vantagens a economia de espaço físico e reunião de documentos, ora dispersos em um mesmo local.

No trabalho de Siqueira (2005) é apresentado o resultado do Grupo Educação e Memória (GEM) da Universidade Federal de Mato Grosso (UFMT), com a organização de fontes escritas e entrevistas por meio da criação de um banco de dados, banco de vozes e banco de fontes, e gerando CD's que integraram um conjunto de fontes privadas, publicas e familiares. Essas ações visaram facilitar o acesso aos pesquisadores sobre a História da Educação daquele estado. Na mesma linha da preservação de fontes, identificou-se o trabalho de Souza (2013), que aponta como imprescindível o debate sobre a conservação e proteção documental como condição para o desenvolvimento do patrimônio educativo do país.

Em consonância às reflexões promovidas por Souza (2013), o trabalho de Silva (2011) também aborda a importância da preservação de documentos de arquivos escolares, apontando como urgente a intervenção de áreas como Arquivologia e Ciências da Informação junto aos pesquisadores da História e História da Educação. O diálogo e o trabalho interdisciplinar entre esses campos se fazem necessário à busca de meios atualizados para a salvaguarda e disponibilização documental eficiente. Essa perspectiva sobre os documentos escolares também foi identificada no trabalho de Fernandes (2010), o qual aponta a fragilidade de recursos tecnológicos e humanos para as atividades de arquivamento e preservação documental nas escolas, comprometendo a recuperação de memórias históricas. Esse autor destaca a urgente necessidade de digitalização do acervo dessas instituições. Por óbvio, essa ação envolve a questão da gestão escolar, aspecto presente na produção de Toschi e Rodrigues (2003), onde é exposto o trabalho de elaboração de um CD com materiais audiovisuais para as disciplinas de História da Educação e Prática de Ensino. O mesmo conteúdo foi utilizado para a criação, pelos autores, de um museu virtual sobre educação. As fontes para o estudo de Toschi e Rodrigues (2003) foram coletadas a partir do ambiente de escolas nas cidades de Goiânia, Anápolis, Jataí e Catalão. Em suas reflexões, os autores afirmam a necessidade de articulação entre as dimensões acadêmica, técnica e de gestão.

Voltado também para a realidade das escolas, Pereira (2011) trabalhou as fontes documentais para a História da Educação, empregando fontes diversas, entre elas as de gênero denominado "informático", as quais são constituídas de CDs, DVDs e disquetes, buscando classificar e hierarquizar o conteúdo encontrado, a fim de constituir uma memória da educação básica pública no Distrito Federal. Essa autora também criou um modelo de processo de digitalização para a elaboração de acervo documental. Esse recurso também foi empregado no trabalho de Soares, Braga e Lima (2015), que apresentam a digitalização de documentos referentes à educação durante o regime militar. O processo foi desenvolvido pela classificação, digitalização, armazenamento e disponibilização, por meio de um repositório digital. Esse mesmo meio tecnológico foi utilizado por Louveira e Ferro (2013). Essas autoras elaboraram um banco de dados fotográfico de crianças e infância do sul do Mato Grosso, criando um catálogo denominado "FotoMemo", o qual foi disponibilizado para uso por meio de CDs e DVDs. A mídia CD também foi adotada no trabalho de Werle (2007) como forma de apoio à pesquisa de identidade e história institucional, discutindo a digitalização e a organização em CDs de documentos que permitam a reconstrução da história de instituições escolares.

No trabalho de Góes (2008) é apresentado um banco de dados sobre a História da Educação, relativo a produções em nível stricto sensu de instituições de ensino soteropolitanas, com foco a reduzir o tempo de busca pelos pesquisadores, além de garantir a integridade e a preservação de acervo, e o acesso ao público. Também sobre a região nordeste, Andrade (2016) apresenta a experiência da criação de um repositório digital com fontes de História da Educação sobre a cidade de Bananeiras, no Estado da Paraíba, com o objetivo de permitir o compartilhamento de documentos para fins de pesquisa. 
Por fim, identificou-se os trabalhos de Piotrowski (2012) e Pettersson et al (2016) que empregaram o processamento de linguagem natural na extração de informações para a análise de documentos históricos, permitindo ampliar a descoberta de informações de forma automatizada. Essa tecnologia viabilizou a detecção de padrões em documentação escrita em Inglês, no período de 1504 a 1900, indicando ocorrências sobre a educação nas fontes investigadas.

\section{Conclusão}

Embora não se tenha identificado um padrão sobre determinada tecnologia a ser empregada no tratamento de fontes para a História da Educação, foi possível constatar que a digitalização tem sido o recurso mais utilizado nos trabalhos dos pesquisadores desse campo. É possível inferir-se que isso ocorre em razão do objetivo, indicado como urgente pelos autores, de atendimento à necessária preservação de acervos. No entanto, cabe ressaltar que o meio ou a mídia utilizada para a digitalização em um determinado período pode inviabilizar o resgate da fonte em outro momento. É necessário considerar que a atualização tecnológica, atualmente, ocorre de forma dinâmica e envolve os próprios equipamentos para o acesso, a visualização e a leitura desse acervo.

Observou-se que há uma preocupação dos autores em construir um acervo a partir das suas pesquisas. A disponibilização dos documentos coletados ocorre, com maior frequência, por meio de CD ou DVD. Há, ainda, autores que investiram na elaboração de banco de dados para catalogar os seus trabalhos. Com menor incidência, os pesquisadores implementaram sites ou repositórios digitais para a distribuição de conteúdos. Esse cenário indica a preocupação em não apenas digitalizar as fontes, mas, também, em desenvolver meios de busca e consulta às informações. Em acréscimo, Piotrowski (2012), alerta que os pesquisadores da História da Educação não podem se deter apenas em catalogar, mas devem, também, buscar informações por meio do processamento de linguagem natural, o que permitirá o uso do computador para o entendimento e interpretação de linguagem humana.

Assim, torna-se necessário o domínio de tais técnicas por parte do pesquisador, não para a criação dos recursos tecnológicos, mas para a sua utilização no cotidiano das pesquisas. Isso permitirá a preservação documental e o avanço científico relativo às pesquisas de História da Educação, democratizando o acesso à comunidade acadêmica. Esse é um caminho que, embora já venha sendo percorrido por diversos pesquisadores, ainda demanda maior interesse para a ampliação do uso das novas tecnologias. Como sugerido por Faria Filho (2000), há a necessidade de criação de um repositório virtual nacional da História da Educação, o qual além de unificar o acesso pelos pesquisadores, subsidiaria as pesquisas históricas, permitiria a conservação dos dados e as buscas integradas a uma base de conhecimento.

\section{Agradecimentos}

Registramos nossos meus agradecimentos à Universidade Tiradentes pelo apoio e bolsa que possibilitaram a execução deste trabalho, ao CNPq pelo apoio institucional e fomento ao Projeto de Pesquisa coordenado pela Profa. Ester Fraga Vilas-Bôas Carvalho do Nascimento, intitulado Rede Brasil, Inglaterra e Portugal: circulação de impressos protestantes e outros impressos pedagógicos durante os Oitocentos (Edital MCT/CNPq 02/2009; Edital Universal CNPq 14/2011 Faixa B; Edital Universal, 2015; Bolsa de Produtividade em Educação/CNPq 2012, 2015, 2018).

\section{Referências}

AMORIM, Eliane Dutra. Arquivos, pesquisa e as novas tecnologias. In: FARIA FILHO, Luciano Mendes. (Org.). Arquivos, fontes e nova tecnologia: questões para a história da educação. Campinas: Autores Associados/ Bragança Paulista: Universidade São Francisco, 2000, p. 89-99. 
ANDRADE, Vivian Galdino. A experiência de criação de um repositório digital como fonte de pesquisa para a história da educação de Bananeiras. Revista de História e Historiografia da Educação, v. 1, n. 2, p. 266-284, 2017. https://doi.org/10.5380/rhhe.v1i2.50376

ANPED. GT02 - História da Educação. Disponível em: http://www.anped.org.br/grupos-detrabalho/gt02-\%E2\%80\%93-hist\%C3\%B3ria-da-educa\%C3\%A7\%C3\%A3o. Acesso em 05 jul. 2018.

BLANCHE, Martin Terre; DURRHEIM, Kevin; PAINTER, Desmond. Research in practice: applied methods for the social sciences. 2. ed. Cape Town: University of Cape Town Press, 2006.

BOAVENTURA, Edivaldo M. Metodologia da pesquisa: monografia, dissertação, tese. São Paulo: Atlas, 2004.

BONATO, Nailda Marinho da costa. O uso das fontes documentais na pesquisa em história da educação e as novas tecnologias. Acervo, v.17, n. 2 jul-dez, p.85-110, 2011.

CABRAL, Ana Maria Rezende. Tecnologia Digital em bibliotecas e arquivos. Transinformação, Campinas, v.14, n.2, p.167-177, 2002. https://doi.org/10.1590/S010337862002000200006

CHARTIER, Roger. A história cultural: entre práticas e representações. Lisboa: Difel, 1990.

FARIA FILHO, Luciano Mendes. (Org.). Arquivos, fontes e nova tecnologia: questões para a história da educação. Campinas: Autores Associados/ Bragança Paulista: Universidade São Francisco, 2000.

FERNANDES, Lincoln Christian. Arquivos escolares e memória: novas perspectivas da pesquisa histórica a partir das novas tecnologias da informação. In: X Encontro de História de Mato Grosso do Sul. Três Lagoas, UFMS, 2010, p. 1058-1069.

GONDRA, José G. A leveza dos bits. In: FARIAS FILHO, Luciano Mendes. Arquivos, fontes e novas tecnologias: questões para a História da Educação. Campinas: Editores Associados, 2000, p.3-17.

GALVÃO, Ana Maria de Oliveira; LOPES, Eliane Marta Teixeira. Território plural: a pesquisa em história da educação. São Paulo: Atica, 2011.

GIL, Antonio Carlos. Como elaborar projetos de pesquisa. 5. ed. São Paulo: Atlas, 2010.

GÓES, Camila Magalhães. A produção historiográfica educacional das ies soteropolitanas: arquivos e dados. In: Anais V Congresso Brasileiro de História da Educação. Aracaju, UFS/UNIT, 2008, p. 1-11.

HABIB, Mamum; PATHIK, Bischwajit Banik; MARYAM, Hafsa. Research methodoogy: contemporary practices. Newcastle upon Tyne: Cambridge Scholars Publishing, 2014.

KOTHARI, Chakravanti Rajagopalachari. Research methodology: methods and techniques. New Delhi: New Age International, 2004. 
LE GOFF, Jacques. História e memória. Tradução: Irene Ferreira, Bernardo Leitão, Suzana Ferreira Borges. Campinas: Editora UNICAMP, 1996.

LOUVEIRA, Andreína de Melo; FERRO, Maria Eduarda. CONSTITUIÇÃO DE UM CATÁLOGO FOTOGRÁFICO DIGITAL COMO FERRAMENTA DE PESQUISA EM HISTÓRIA DA EDUCAÇÃO. In: Anais VII Congresso Brasileiro de História da Educação. Cuiabá, UFMT, 2013, p. 1-14.

LOPES, Ivone Goulart et al. O fio da história-nastrilhas de ouropreto do oeste-ro. Vitrais da memória de professores e escolas. In: Anais do XI SeminarioInternacional de La Red Estrado. Mexico: UPN, 2016, p. 1-17.

PENA, M. G.; SILVA, A. C. A digitalização de documentos históricos e a gestão eletrônica de documentos para disponibilização online. Saber Digital, Valença, v. 1, n. 1, p. 85-102, 2008.

PERDIGÃO, Dulce Mantella; HERLINGER, Maximiliano; WHITE, Oriana Monarca. (Org). Teoria e prática da pesquisa aplicada. Rio de Janeiro: Elsevier, 2012.

PEREIRA, Aracy Roza Sampaio. Fontes documentais da história da educação escolar no Distrito Federal (1956-1960). Monográfia (Licenciatura em Pedagogia) - Faculdade de Educação, Universidade de Brasilia, Distrito Federal, p. 77, 2011.

PETTERSSON, Eva et al. HistSearch-Implementation and Evaluation of a Web-based Tool for Automatic Information Extraction from Historical Text. In: HistoInformatics@ DH. Krakow: Pedagogical University in Krakow, 2016. p.25-36.

PIOTROWSKI, Michael. Natural language processing for historical texts. Synthesis Lectures on Human Language Technologies, v.5, n.2, p.1-157, 2012. https://doi.org/10.2200/S00436ED1V01Y201207HLT017

SEVERINO, Antônio Joaquim. Metodologia do trabalho científico. 27.ed. São Paulo: Cortez, 2007.

SIQUEIRA, Elizabeth Madureira. Reconstruindo arquivos escolares: a experiência do GEM/MT. Revista Brasileira de História da Educação. Maringa, v. 5, n.2[10], 2005, p.123-152.

SILVA, Eva Cristina Leite da. Mapeamento dos arquivos escolares: história, memória e preservação de documentos. Ágora, Florianópolis, v.21, n.42, 2011, p.111-125.

SOARES, Irvin Bezerra; BRAGA, Mirella de Almeida; LIMA, Izabel França de. Digitalização de Documentos: disponibilizando as fichas do DOPS do período da ditadura militar na Internet. InterScientia. João Pessoa. v.3, n.2, 2015, p.17-28.

SOUZA, Rosa Fátima de. Preservação do Patrimônio Histórico Escolar no Brasil: notas para um debate. Revista Linhas. Florianópolis, v.14, n.26, 2013. p.199-221. https://doi.org/10.5965/1984723814262013199 
TOSHI, Mirza Seabra; RODRIGUES, Maria Emília de Castro. Infovias e educação. Educação e Pesquisa. São Paulo, v.29, n.2, 2003, p.313-326. https://doi.org/10.1590/S1517$\underline{97022003000200009}$

TUCHMAN, Barbara W. A prática da história. Tradução Waltersir Dutra. Rio de Janeiro: José Olympio, 1991.

Vieira, Alboni Marisa DudequePianovski. Os documentos microfilmados e/ou digitalizados como fonte para o estudo da história da educação: avanços e possibilidades. In: Anais do VI Congresso Brasileiro de História da Educação. Vila Velha: UFES, 2011, p. 1-11.

WAZLAWICK, Raul Sidnei. História da Computação. Rio de Janeiro: Elsevier, 2016.

WERLE, Flavia Obino Corrêa. Cd-rom como apoio na pesquisa sobre a identidade e a história institucional. Educação Unisinos. São Leopoldo, v.11, v.2, 2007, p.111-120. 